\title{
Arthroscopic stabilisation of an acute acromioclavicular dislocation grade III in a patient with ectopic insertion of the pectoralis minor: technical considerations
}

\author{
Alvaro Minuesa-Asensio • Carmen Barrio-Asensio • \\ Ignacio González-Gómez · Jorge Murillo-González
}

Received: 28 March 2014 / Accepted: 24 November 2014 / Published online: 2 December 2014

(C) European Society of Sports Traumatology, Knee Surgery, Arthroscopy (ESSKA) 2014

\begin{abstract}
The different approaches used in arthroscopic stabilisation of the acromioclavicular joint are well known. However, and despite a great incidence of ectopic pectoralis minor insertion, an alternative choice for the use of arthroscopic portal has not being sufficiently described. Here, we describe a case of acute acromioclavicular dislocation grade III. The arthroscopic stabilisation was achieved using the TightRope (Arthrex, Naples, USA) implant. Through this technique, the approach to the articular portion of the coracoid process can be made intra-articularly or from the subacromial space. We accessed intra-articularly, by opening the rotator interval to reach the coracoid process from the joint cavity. After opening the rotator interval, an ectopic insertion of the pectoralis minor was observed. The choice of approach of the coracoid process from the subacromial space would have complicated the intervention, making it necessary to sever the ectopic tendon to complete the technique, lengthening the surgical time and increasing the chance of complications. For this reason, the use of a standard posterior portal providing intra-articular arthroscopic access through the rotator interval is recommended since the aforementioned anatomical variation is not infrequent.
\end{abstract}

Level of evidence Therapeutic studies-investigating the results of treatment, Level V.

A. Minuesa-Asensio · I. González-Gómez

Department of Traumatology, University Hospital of Guadalajara, Guadalajara, Spain

C. Barrio-Asensio · J. Murillo-González ( $\square)$

Department of Anatomy and Human Embryology, School

of Medicine, Complutense University of Madrid, 28040 Madrid,

Spain

e-mail: jmurillo@med.ucm.es
Keywords Acromioclavicular joint - Arthroscopic stabilisation · Pectoralis minor $\cdot$ TightRope ${ }^{\circledR}$ technique

\section{Introduction}

The different approaches used in arthroscopic stabilisation of the acromioclavicular joint are well known. However, and despite a great incidence of ectopic pectoralis minor insertion (10-15\% of cases) [2, 3, 9], an alternative choice for the use of arthroscopic portal has not being sufficiently described. This anatomical variation, well known to surgeons, is often not taken into account because their clinical implications are minimal in most cases. However, this anatomical variation must be taken into account when choosing the arthroscopic approach to avoid potential complications.

In this report, a case of arthroscopic stabilisation of acromioclavicular dislocation on a left shoulder with anatomical variation in the insertion of the pectoralis minor is presented.

\section{Case report}

A 36-year-old male was brought to the emergency service after a bicycle fall with an acute grade III acromioclavicular joint dislocation. A surgical stabilisation of the joint was performed using the TightRope ${ }^{\mathrm{TM}}$ (Arthrex, Naples, USA) technique. The intra-articular approach was performed using a posterior portal to view the area and anterosuperior portal for the introduction of the guide. The exposure of the coracoid process allowed the detection, in the entire upper face of the horizontal portion, of an anatomical variation in the insertion of the pectoralis minor's tendon (Fig. 1). It 


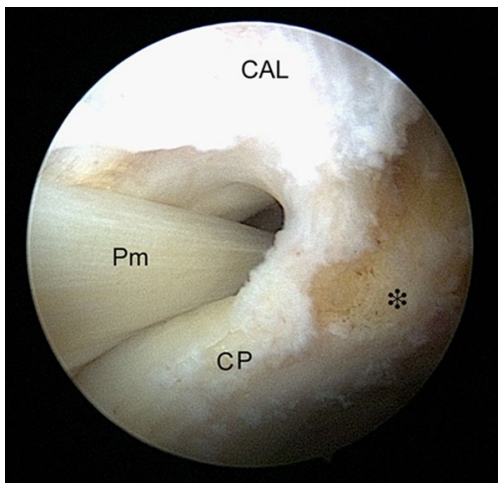

Fig. 1 Case illustration: the entire tendon of the pectoralis minor is observed passing over the horizontal portion of the coracoid process, through the coracoacromial ligament which is fenestrated (the asterisk is located at the tip of coracoid process). $C A L$ coracoacromial ligament, $C P$ coracoid process, $P m$ pectoralis minor tendon

was decided to continue respecting the pectoralis minor's tendon and completing the procedure without incident, obtaining a stable acromioclavicular joint.

\section{Discussion}

Acromioclavicular dislocation is a relatively common injury during the practice of a number of sports. Traditional stabilisation techniques have recently been giving way to arthroscopic techniques becoming more popular in the treatment of these injuries. In the present case, the TightRope ${ }^{\mathrm{TM}}$ system was used (Arthrex, Naples, USA). This system uses a simple arthroscopic technique that has proved to be effective in the acute stabilisation of the acromioclavicular joint. In this technique, the approach to the articular portion of the coracoid process can be made intra-articularly, by opening the rotator interval, or from the subacromial space. In both approaches, the portals used are relatively safe, since they are located lateral to the coracoid process, allowing most of the work to be performed at a safe distance from the neurovascular structures subject to injure. The choice to use the intra-articular approach in the case reported here facilitated the technique allowing for the preservation of the ectopic insertion of the pectoralis minor. The subacromial approach would have required the exposure of the coracoid, and surely, oblige the section of the pectoralis minor's tendon. Although it has been found in other surgical procedures, section of the pectoralis minor is not a discernible functional deficit [1], this would have complicated the technique lengthening the surgical time and increasing the chance of complications.

The pectoralis minor, in most cases, is located deep with respect to the pectoralis major, extending from the scapula to the rib cage. It originates from three meaty fingers of the external face of the third, fourth and fifth ribs near their costochondral joints. The muscle has its insertion in the horizontal portion of the coracoid process in the anterior half of the medial edge and near its upper face. Variations in insertion have been described: surpassing its tendon from the coracoid process to attach to the coracoacromial ligament, the glenoid rim, the supraspinatus tendon, the capsule of the glenohumeral joint, the humeral tubercles, etc. [2-9]. Le Double [3] observed anomalies in the pectoralis minor insertion in $15 \%$ of 106 dissections and was the first to classify them by describing three types. Weinstabl et al. [9] performed an anatomical study of 126 shoulders finding continuity between the coracohumeral ligament and the pectoralis minor and pectoralis minor fibres passing over the coracoid process in $16 \%$ of cases. Homsi et al. [2] used ultrasounds of 606 shoulders (303 patients) and observed abnormalities in the insertion of the pectoralis minor in $9.57 \%$ of cases.

This anatomical variation, which is well known to surgeons, is often not taken into account because their clinical implications are minimal in most cases. However, the possible presence of an ectopic insertion of the pectoralis minor in addition to possible damage to the neighbouring neurovascular elements must be taken into account when choosing the arthroscopic portal to stabilise the dislocation of the acromioclavicular joint. The subacromial approach would have complicated the technique by lengthening the surgical time and increasing the chance of complications. For this reason, the intra-articular approach is recommended in order to facilitate the performance of the technique resolving the possible presence of this anatomical variation and avoiding further complications.

Acknowledgments The authors thank Javier Catón-Vázquez for translating the manuscript. We also thank the Proof-Reading-Service UK, for the English review of this manuscript.

\section{References}

1. De Beer JF, Roberts C (2010) Glenoid bone defects-open latarjet with congruent arc modification. Orthop Clin N Am 41(3):407-415

2. Homsi C, Rodrigues MB, Silva JJ, Stump X, Morvan G (2003) Anomalous insertion of the pectoralis minor muscle: ultrasound findings. J Radiol 84(9):1007-1011

3. Le Double AF (1987) Traitè des variations du système musculaire de l'homme et de leur signification au point de vue de l'anthropologie zoologique. In: Libraire C. Rerinwald (ed). Schleicher Frères, Paris pp 252-255

4. Lee SJ, Ha DH, Lee SM (2010) Unusual variation of the rotator interval: insertional abnormality of the pectoralis minor tendon and absence of the coracohumeral ligament. Skelet Radiol 39(12):1205-1209

5. Samuel P, Blanchard JP (1984) Syndrome de la coiffe des rotateurs par anomalie d'insertion du petit pectoral [Rotator cuff syndrome caused by an anomaly of the insertion of the pectoralis minor]. Rev Chir Orthop Reparatrice Appar Mot 70(5):401-404 
6. Testut L (1884) Les anomalies musculaires chez l'homme, expliquées par l'anatomie comparée: leur importance en anthropologie.In: G. Masson (ed). Paris, pp 34-44. https://archive.org/detail s/lesanomaliesmusc00testuoft

7. Tubbs RS, Oakes WJ, Salter EG (2005) Unusual attachment of the pectoralis minor muscle. Clin Anat 18(4):302-304
8. Uzel AP, Bertino R, Caix P, Boileau P (2008) Bilateral variation of the pectoralis minor muscle discovered during practical dissection. Surg Radiol Anat 30(8):679-682

9. Weinstabl R, Hertz H, Firbas W (1986) Zusammenhang des ligamentum coracoglenoidale mit dem musculus pectoralis minor. Acta Anat 125:126-131 